This is the Author Accepted Manuscript of an article: Niina Khan, Jahangir Khan, Leo-Pekka Lyytikäinen, Terho Lehtimäki, Jari Laurikka \& Niku Oksala (2020) Serum apolipoprotein A-I concentration differs in coronary and peripheral artery disease, Scandinavian Journal of Clinical and Laboratory Investigation. Published online 03 Jun 2020 by Taylor \& Francis. The final version available at: http://dx.doi.org/10.1080/00365513.2020.1746974

Title page

\title{
Serum Apolipoprotein A-I Concentration Differs in Coronary and Peripheral Artery Disease
}

Running head: Apo A-I in Atherosclerotic Disease

\author{
Niina Khan ${ }^{\mathrm{a}}$, Jahangir Khan ${ }^{\mathrm{b}}$, Leo-Pekka Lyytikäinen ${ }^{\mathrm{c}, \mathrm{d}}$, Terho \\ Lehtimäki $^{c, d}$, Jari Laurikka ${ }^{\text {b,c }}$, Niku Oksala ${ }^{\mathrm{a}, \mathrm{c}}$
}

${ }^{a}$ Center for Vascular Surgery and Interventional Radiology, Tampere University Hospital, Tampere, Finland; ${ }^{b}$ Department of Cardio-Thoracic Surgery, Heart Hospital, Tampere University Hospital, Tampere, Finland; ' Finnish Cardiovascular Research Center - Tampere, Faculty of Medicine and Health Technology, Tampere University, Tampere, Finland;

${ }^{\mathrm{d}}$ Department of Clinical Chemistry, Fimlab Laboratories, Tampere, Finland

E-mail addresses for authors:

Niina Khan: khan.niina@gmail.com

Jahangir Khan: jahangir.khan@saunalahti.fi

Leo-Pekka Lyytikainen: leo-pekka.lyytikainen@tuni.fi

Terho Lehtimäki: terho.lehtimaki@pshp.fi

Jari Laurikka: jari.laurikka@tuni.fi

Niku Oksala: niku.oksala@tuni.fi

Word count: abstract: 243; main text: 1891; entire manuscript: 3671

Declaration of conflicting interests: The authors report no conflicts of interest.

Corresponding author: Niina Khan, Center for Vascular Surgery and Interventional Radiology, Tampere University Hospital, Teiskontie 35 PO BOX 2000, 33521 Tampere, Finland; tel +358 311611; email: khan.niina@gmail.com 


\title{
Serum Apolipoprotein A-I Concentration Differs in Coronary and Peripheral Artery Disease
}

\begin{abstract}
Coronary artery and peripheral artery disease represent different clinical outcomes of atherosclerosis and despite sharing common risk factors the ultimate reasons determining disease presentation are still unclear. The present study sought to define and compare the serum lipid and apolipoprotein profiles of patients undergoing coronary artery bypass grafting and those treated invasively for symptomatic lower extremity peripheral artery disease. Altogether 218 coronary and 280 peripheral artery disease patients treated between 2013 and 2014 in the Tampere University Hospital, Tampere, Finland, with available lipid measurements within two years prior to the intervention were retrospectively analysed. The Extended Friedewald formula neural network model was used to obtain apolipoprotein and lipoprotein subfraction values. Patients undergoing coronary artery bypass surgery had a clear male predominance (82\% versus $53 \%$, $\mathrm{p}<0.001$ ), lower median age (69 versus 74 years, $\mathrm{p}<0.001$ ) and a lower prevalence of smoking ( $18 \%$ versus $32 \%, \mathrm{p}=0.001$ ) and pulmonary disease ( $12 \%$ versus $20 \%, \mathrm{p}=0.023$ ) compared to peripheral artery disease patients. There were some differences in the serum lipid profiles between the study groups in the univariable analyses. When controlling for the statistically significant differences in age, sex, urgency of treatment and comorbidities between the groups in a multivariable logistic regression model, higher serum concentrations of apolipoprotein A-I were significantly and independently associated with coronary artery disease (OR 1.11 for $0.01 \mathrm{~g} / \mathrm{L}$ increase, $\mathrm{p}=0.044$ ). In conclusion, patients undergoing coronary artery bypass grafting appear to have higher apolipoprotein A-I levels when compared to patients treated for peripheral artery disease.
\end{abstract}

Keywords: Atherosclerosis, Coronary artery bypass grafting, Coronary artery disease, Lipids, Peripheral arterial disease 


\section{Introduction}

Atherosclerosis is a leading cause of morbidity and health-care costs. Peripheral artery disease (PAD) and coronary artery disease (CAD) represent different manifestations of atherosclerosis and while coexisting conditions are possible, in most patients either $\mathrm{PAD}$ or $\mathrm{CAD}$ is dominant. The reasons behind varying disease presentation remain unclear. Risk factors for PAD and CAD are similar though their relative importance differs [1]. Dyslipidaemia is clearly associated with the development and progression of atherosclerosis. Particularly high serum total cholesterol (TC) and low-density lipoprotein cholesterol (LDL-C) have been considered important factors in the emergence and progression of PAD and CAD and lipid-lowering medical therapy is a cornerstone of conservative treatment for the diseases [2-6].

Contemporary serum lipid parameters such as apolipoproteins and lipoprotein subfractions are increasingly recognised as complementary or even superior to the traditional lipid markers in cardiovascular risk assessment. Some guidelines recommend apolipoprotein B (apoB) and/or non-high-density lipoprotein cholesterol (non-HDL-C) measurements [7,8] and lipoprotein(a) - an apolipoprotein A (apoA) particle bound to a low-density lipoprotein cholesterol (LDL-C) -like particle - was discovered to be associated with PAD and its progression [9-13]. While the direct methods for obtaining apolipoprotein and lipoprotein subfraction concentrations are resource consuming, these can be estimated from traditional Friedewald inputs - serum total cholesterol (TC), triglycerides (TG), and HDL-C - by applying a neural network model, the extended Friedewald formula (EFW), accurately, swiftly and with lower costs [14].

The hypothesis for the present study was that the serum lipid and apolipoprotein profile is associated with the clinical manifestation of atherosclerosis. The serum lipid profiles were compared between patients invasively treated for PAD and those undergoing coronary artery surgery. Novel EFW lipid parameters were included. 


\section{Materials and methods}

\section{Patients and methods}

The present study is a retrospective analysis comparing 218 patients that underwent isolated coronary artery bypass surgery and 280 patients that received invasive treatment - involving surgical, endovascular, or hybrid procedures - for peripheral lower extremity artery disease at Tampere University Hospital and Tampere University Heart Hospital between January 2013 and December 2014. The study subjects were identified from institutional prospective databases that contain information on all consecutive patients treated at the clinics. The clinical course of each case was reviewed and all patients whose serum lipid values had been measured within two years prior to the intervention were included in the analyses. The patients' demographic information, relevant medical history including statin use, urgency of the intervention as well as procedural details were recorded. If the same patient underwent treatment more than once during the study period, only the first treatment episode was included.

\section{Lipid Analysis}

Traditional serum lipid values including TC, HDL-C, and triglycerides (TG), were analysed using routine laboratory methods and were obtained from the Fimlab laboratories Ltd database. The median time interval between the lipid analysis and the treatment episode for PAD or CAD was 104 (range 0-728) days. The concentration of LDL-C was estimated by the traditional Friedewald formula [15]. If the serum lipid values of a patient had been measured more than once, the values closest to the index procedure were included. The Extended Friedewald formula -derived parameters - verylow-density lipoprotein triglycerides (EFW-VLDL-TG), intermediate-density lipoprotein cholesterol (EFW-IDL-C), low-density lipoprotein-cholesterol (EFW-LDLC), high-density lipoprotein-cholesterol subfraction 2 (EFW-HDL2), apolipoprotein A-I (EFW-ApoA-I) and apolipoprotein B (EFW-ApoB) - were determined as described by Niemi et al by using traditional serum lipid parameters as inputs [14]. 


\section{Statistical Analysis}

The study is of a descriptive nature and thus no prior power analysis was performed. All statistical testing was carried out with SPSS version 16.0 for Windows. The univariable comparisons of patient characteristics and serum lipid values between CAD and PAD patients were done using the Mann-Whitney U-test for noncategorical scale variables and the Chi-Square and the Fisher's exact test for categorical data. To control for the effect of group differences in the lipid values, statistically significant differences in the baseline characteristics - including age, sex, statin use, smoking, urgency of treatment and the prevalence of chronic pulmonary disease - were included in a multivariable logistic regression analysis. The statistical significance was set at $\mathrm{p}<0.05$. The study was performed according to the ethical principles of The Helsinki Declaration. Institutional review board approval was obtained. 


\section{Results}

Altogether 218 patients treated for CAD and 280 patients treated for lower extremity PAD were included in the study. Critical limb threatening ischemia was present in $53.9 \%$ of PAD patients and $50.9 \%$ of patients that underwent coronary surgery were treated for acute coronary syndrome. The patients treated for PAD underwent operations comprising endarterectomies in $20 \%$, bypasses in $26 \%$, patch angioplasties in $10 \%$, and/or hybrid or isolated endovascular procedures in $61 \%$ of cases. The patients undergoing coronary surgery were operated through standard median sternotomy and the median number of bypasses performed was three (range 1-7). The demographic information of the study groups is shown in Table 1 . The patients undergoing coronary artery bypass grafting were more frequently male, somewhat younger, had lower rates of smoking and pulmonary diseases and higher rates of statin use. Also, urgent or emergency treatment was more common in patients undergoing coronary artery bypass grafting.

In univariable analyses, the traditional lipid parameter distributions were similar between the groups aside from HDL-C, which was more favourable in PAD patients (Table 2). Similarly, there were no significant differences between the concentrations of EFW-VLDL-TG, EFW-IDL-C or EFW-LDL-C, but the EFW-HDL2-subfraction concentrations and EFW-ApoA-I as well as the EFW-ApoB/ApoA-I were statistically significantly more favourable in patients with PAD (Table 2). Overall, EFW-ApoA-I levels were significantly higher in females when compared to males, 1.56 versus 1.33 $\mathrm{g} / \mathrm{L}, \mathrm{p}<0.001$, and in patients aged at least 70 years when compared to younger patients, 1.45 versus $1.35 \mathrm{~g} / \mathrm{L}, \mathrm{p}=0.001$, respectively. In the multivariable analysis (Table 3) older age and smoking were independently associated with a decreased risk of CAD as opposed to PAD. Conversely, statin use, male sex and a higher EFW-ApoA-I concentration were independently associated with a higher risk of CAD rather than PAD. 


\section{Discussion}

The anatomical presentations of and the clinical syndromes caused by atherosclerosis are multiform and the factors influencing its manifestation are not completely understood. Atherosclerotic clinical conditions, such as PAD and CAD, share several risk factors though the gravity of each is variable. Serum lipid disorders are clearly associated with atherosclerosis. The present study sought to discern variances in the serum lipid profiles, including modern lipid parameters, between PAD and CAD patients and suggests an interesting difference between the atherosclerotic phenotypes.

The study included patients undergoing invasive treatment for PAD and those undergoing coronary surgery for CAD, essentially representing advanced stages of symptomatic atherosclerotic disease in both groups. The proportion of patients undergoing urgent or emergency coronary surgery in the present study is somewhat high but other series have also reported a similar change in the ratio possibly reflecting an ongoing trend towards percutaneous procedures in the treatment of elective CAD cases $[16,17]$. Overall, the presentation and disease severity of PAD patients in the current study is comparable to that in the literature [18]. As in earlier studies, there were some differences in the PAD and CAD patient cohorts. Firstly, PAD patients tended to be older which can be explained by the generally later clinical onset of the disease $[1,19]$. Secondly, the prevalence of smoking and pulmonary disease was higher in the PAD group. This is in line with previous studies as smoking is one of the most potent risk factors for PAD but its significance in CAD is less pronounced [1,20,21]. Thirdly, there was a clear male predominance in the CAD cohort, possibly explained by a tendency for selecting non-surgical revascularisation methods for female CAD patients, as elsewhere [16]. Male sex is a risk factor for both symptomatic CAD and PAD but sex differences in the incidence and prevalence of the latter are less pronounced $[1,19,22]$. Albeit statin medication is advocated for all symptomatic CAD and PAD patients, the rate of statin use in the present study was greater in CAD patients compared to PAD patients, which agrees with previous literature [23-27]. Although the included study groups somewhat differ, all in all, they should accurately reflect real life patients and clinical practice.

With regards to the lipid parameters, the present study found disease specific differences in lipid values between CAD and PAD patients. Of the traditional lipid 
parameters, HDL-C values were higher in patients with PAD despite a lower use of statins, which tend to increase both HDL-C and ApoA-I levels [28], and an older median age, which has been found to be associated with a decrease in HDL-C concentrations [29]. Similar findings have been reported by other authors as well $[19,20]$. The difference may be partly explained by the greater proportion of females in the PAD cohort and was not evident after multivariable adjusting. Of the contemporary lipid parameters, EFW-ApoA-I, EFW-ApoB/ApoA-I ratio, and EFW-HDL 2 differed between the patient cohorts and were more favourable in the PAD group. However, in the multivariable analysis, higher EFW-ApoA-I was independently associated with CAD, which is a novel finding. Previously, ApoB/ApoA-I has been found to be an effective predictor of CAD in obese patients and the inclusion of EFW-derived apolipoprotein parameters has improved cardiovascular risk prediction [30,31]. In disease-free individuals, low levels of serum ApoA-I have predicted the development of or death due to both CAD and PAD [5,32]. In patients with established cardiovascular disease, low ApoA-I levels have been associated with a higher incidence of cardiovascular events and death [33,34], critical limb ischemia [35], as well as worse prognosis following coronary artery bypass surgery [36]. Furthermore, an inverse relationship between PAD and HDL-C as well as lower HDL-C levels in CAD patients compared to PAD patients have been reported in earlier literature [19,20]. A study by Tunstall-Pedoe et al [5] additionally discovered serum TC to be more predictive of CAD compared to PAD but failed to ascertain other differences in lipid profiles. Taken together, the combined evidence regarding the potential role of ApoA-I in determining the phenotype of atherosclerosis is thus far inconclusive. However, this interesting and novel finding warrants further research.

The main limitations of the study include its retrospective nature, single centre setting and restricted cohort size. The inclusion of patients with preceding lipid measurements may have caused patient selection. Furthermore, possible concomitant CAD or PAD was not controlled for, but the treatment was given for the disease that was clinically relevant at the time. The differing baseline characteristics of PAD and CAD patients are also a potential source of bias in univariable analyses. However, these were accounted for in the multivariable analysis. All in all, the results of the current study must be viewed with caution, but they suggest previously unrecognised lipid profile differences between PAD and CAD patients. 
In conclusion, patients undergoing coronary artery bypass surgery appear to have higher apolipoprotein A-I levels when compared to patients treated for peripheral artery disease. Other differences in the serum lipid profiles are likely to be explained by variance in the baseline characteristics such as age and sex.

Declaration of interest statement: The authors report no conflicts of interest.

\section{Funding}

This work was supported by the Competitive Research Funding of the Tampere University Hospital (under Grant X51001 for T.L. and N.O.), the Emil Aaltonen Foundation (T.L., N.O., and J.K.), the Academy of Finland (Grant 286284), the Finnish Foundation for Cardiovascular Research, the Yrjö Jahnsson Foundation, European Union 7th Framework Program (grant 201668 for AtheroRemo), and the EU Horizon 2020 (grant 755320 for TAXINOMISIS). 


\section{Tables}

Table 1. Demographic data of study groups. Statistically significant differences between groups are highlighted in bold.

\begin{tabular}{llll}
\hline & Coronary bypass surgery & Lower extremity artery & $\mathrm{p}^{-}$ \\
& patients & disease & value \\
\hline Number of patients & 218 & 280 & \\
Male (\%) & $\mathbf{8 2}$ & 53 & $<\mathbf{0 . 0 0 1}$ \\
Median age (years, range) & $\mathbf{6 9}(\mathbf{4 2 - 8 9 )}$ & $\mathbf{7 4}(\mathbf{4 5 - 9 5 )}$ & $<\mathbf{0 . 0 0 1}$ \\
Diabetes (\%) & 38 & 40 & 0.645 \\
Statin use (\%) & $\mathbf{7 9}$ & $\mathbf{5 1}$ & $<\mathbf{0 . 0 0 1}$ \\
Hypertension (\%) & 74 & 75 & 0.771 \\
Smoking (\%) & $\mathbf{1 8}$ & 32 & $\mathbf{0 . 0 0 1}$ \\
Urgent treatment (\%) & $\mathbf{5 1}$ & $\mathbf{2 5}$ & $<\mathbf{0 . 0 0 1}$ \\
Chronic pulmonary & $\mathbf{1 2}$ & $\mathbf{2 0}$ & $\mathbf{0 . 0 2 3}$ \\
disease (\%) & & & \\
\hline
\end{tabular}


Table 2. Serum lipid profiles of patients undergoing isolated coronary artery bypass grafting or interventional treatment for lower extremity artery disease.

\begin{tabular}{|c|c|c|c|}
\hline & $\begin{array}{l}\text { Coronary bypass } \\
\text { surgery patients }\end{array}$ & $\begin{array}{l}\text { Lower extremity artery } \\
\text { disease }\end{array}$ & $\begin{array}{l}\mathrm{p}^{-} \\
\text {value }\end{array}$ \\
\hline Total cholesterol (mmol/L) & 4.2 & 4.5 & 0.353 \\
\hline LDL-cholesterol (mmol/L) & 2.31 & 2.39 & 0.772 \\
\hline HDL-cholesterol (mmol/L) & 1.18 & 1.27 & 0.006 \\
\hline Triglycerides (mmol/L) & 1.27 & 1.34 & 0.824 \\
\hline EFW-VLDL-TG (mmol/L) & 0.73 & 0.76 & 0.993 \\
\hline EFW-IDL (mmol/L) & 0.21 & 0.21 & 0.706 \\
\hline EFW-LDL (mmol/L) & 2.51 & 2.60 & 0.797 \\
\hline $\mathrm{EFW} \mathrm{HDL}_{2}(\mathrm{mmol} / \mathrm{L})$ & 0.69 & 0.77 & 0.004 \\
\hline EFW-ApoA-I (g/L) & 1.37 & 1.46 & 0.006 \\
\hline EFW-ApoB (g/L) & 0.89 & 0.91 & 0.942 \\
\hline EFW-ApoB/ApoA-I & 0.66 & 0.61 & 0.032 \\
\hline
\end{tabular}


Table 3. Multivariable logistic regression analysis including significant preoperative patient characteristics and the serum lipid measurements. The Odds ratios are calculated for having coronary artery disease instead of peripheral artery disease and represent an increase of 0.01 $\mathrm{mmol} / \mathrm{L}$ for cholesterol and triglyceride and $0.01 \mathrm{~g} / \mathrm{L}$ for apolipoprotein levels.

\begin{tabular}{lll}
\hline & Odds Ratio & p-value \\
\hline Male sex & $\mathbf{4 . 0 1}$ & $<\mathbf{0 . 0 0 1}$ \\
Age $\geq \mathbf{7 0}$ & $\mathbf{0 . 5 1}$ & $\mathbf{0 . 0 0 5}$ \\
Statin use & $\mathbf{3 . 9 0}$ & $<\mathbf{0 . 0 0 1}$ \\
Smoking & $\mathbf{0 . 2 6}$ & $<\mathbf{0 . 0 0 1}$ \\
Chronic pulmonary disease & 0.56 & 0.063 \\
Total cholesterol & 0.90 & 0.389 \\
LDL-cholesterol & 1.11 & 0.343 \\
HDL-cholesterol & 0.97 & 0.835 \\
EFW-VLDL-TG & 1.04 & 0.491 \\
EFW-IDL & 1.00 & 0.931 \\
EFW-LDL & 0.97 & 0.389 \\
EFW-HDL2 & 1.06 & 0.209 \\
EFW-ApoA-I & $\mathbf{1 . 1 1}$ & $\mathbf{0 . 0 4 4}$ \\
EFW-ApoB & 1.05 & 0.522 \\
EFW-ApoB/ApoA-I* $0.5-0.7$ & 0.55 & 0.160 \\
EFW-ApoB/ApoA-I* $>0.7$ & 0.41 & 0.207 \\
\hline & & \\
& &
\end{tabular}

*Reference value $<0.5$

Independent variables are highlighted in boldface. 


\section{References}

1. Criqui MH, Aboyans V. Epidemiology of peripheral artery disease. Circ Res 2015;116:15091526.

2. Aboyans V, Ricco J-B, Bartelink MEL, et al. Editor's Choice - 2017 ESC Guidelines on the Diagnosis and Treatment of Peripheral Arterial Diseases, in collaboration with the European Society for Vascular Surgery (ESVS). Eur J Vasc Endovasc J 2018;55:305-368.

3. Montalescot G, Sechtem, U, Achenbach S, et al. 2013 ESC guidelines on the management of stable coronary artery disease. Eur Heart J 2013;34:2949-3003.

4. Ridker PM, Stampfer MJ, Rifai N. Novel risk factors for systemic atherosclerosis: a comparison of C-reactive protein, fibrinogen, homocysteine, lipoprotein(a), and standard cholesterol screening as predictors of peripheral arterial disease. JAMA 2001;285:2481-2485. 5. Tunstall-Pedoe H, Peters SAE, Woodward M, et al. Twenty-Year Predictors of Peripheral Arterial Disease Compared With Coronary Heart Disease in the Scottish Heart Health Extended Cohort (SHHEC). J Am Heart Assoc 2017;6:e005967.

6. Keil U. Coronary artery disease: the role of lipids, hypertension and smoking. Basic Res Cardiol 2000;95 Suppl 1:I52-58.

7. Mach F, Baigent C, Catapano AL, etl al. 2019 ESC/EAS guidelines for the management of dyslipidaemias: lipid modification to reduce cardiovascular risk. Atherosclerosis 2019;290:140205.

8. Morris PB, Ballantyne CM, Birtcher KK, et al. Review of clinical practice guidelines for the management of LDL-related risk. J Am Coll Cardiol 2014;64:196-206.

9. Sniderman AD, Williams K, Contois JH, et al. A meta-analysis of low-density lipoprotein cholesterol, non-high-density lipoprotein cholesterol, and apolipoprotein B as markers of cardiovascular risk. Circ Cardiovasc Qual Outcomes 2011;4:337-345.

10. Walldius G, Jungner I, Aastveit AH, et al. The apoB/apoA-I ratio is better than the cholesterol ratios to estimate the balance between plasma proatherogenic and antiatherogenic lipoproteins and to predict coronary risk. Clin Chem Lab Med 2004;42:1355-1363.

11. Walldius G, Jungner I, Holme I, et al. High apolipoprotein B, low apolipoprotein A-I, and improvement in the prediction of fatal myocardial infarction (AMORIS study): a prospective study. Lancet 2001;358:2026-2033.

12. Laschkolnig A, Kollerits B, Lamina C, et al. Lipoprotein (a) concentrations, apolipoprotein (a) phenotypes, and peripheral arterial disease in three independent cohorts. Cardiovasc Res 2014;103:28-36.

13. Aboyans V, Criqui MH, Denenberg JO, et al. Risk factors for progression of peripheral arterial disease in large and small vessels. Circulation 2006;113:2623-2629. 
14. Niemi J, Mäkinen VP, Heikkonen J, et al. Estimation of VLDL, IDL, LDL, HDL2, apoA-I, and apoB from the Friedewald inputs--apoB and IDL, but not LDL, are associated with mortality in type 1 diabetes. Ann Med 2009;41:451-461.

15. Friedewald WT, Levy RI, Fredrickson DS. Estimation of the concentration of low-density lipoprotein cholesterol in plasma, without use of the preparative ultracentrifuge. Clin Chem 1972;18:499-502.

16. Dalén M, Lund LH, Ivert T, et al. Survival After Coronary Artery Bypass Grafting in Patients With Preoperative Heart Failure and Preserved vs Reduced Ejection Fraction. JAMA Cardiol 2016;1:530-538.

17. Thorsteinsson K, Fonager K, Mérie C, et al. Age-dependent trends in postoperative mortality and preoperative comorbidity in isolated coronary artery bypass surgery: a nationwide study. Eur J Cardiothorac Surg 2016;49:391-397.

18. Soden PA, Zettervall SL, Shean KE, et al. Regional variation in outcomes for lower extremity vascular disease in the Vascular Quality Initiative. J Vasc Surg 2017;66:810-818. 19. Jang SY, Ju EY, Cho SI, et al. Comparison of cardiovascular risk factors for peripheral artery disease and coronary artery disease in the Korean population. Korean Circ J 2013;43:316328.

20. Fowkes FG, Housley E, Riemersma RA, et al. Smoking, lipids, glucose intolerance, and blood pressure as risk factors for peripheral atherosclerosis compared with ischemic heart disease in the Edinburgh Artery Study. Am J Epidemiol 1992;135:331-340.

21. Price JF, Mowbray PI, Lee AJ, et al. Relationship between smoking and cardiovascular risk factors in the development of peripheral arterial disease and coronary artery disease: Edinburgh Artery Study. Eur Heart J 1999;20:344-353.

22. Jousilahti P, Vartiainen E, Tuomilehto J, et al. Sex, age, cardiovascular risk factors, and coronary heart disease: a prospective follow-up study of 14786 middle-aged men and women in Finland. Circulation 1999;99:1165-1172.

23. Chen DC, Armstrong EJ, Singh GD, et al. Adherence to guideline-recommended therapies among patients with diverse manifestations of vascular disease. Vasc Health Risk Manag 2015;11:185-192.

24. DeCarlo C, Scher L, Shariff S, et al. Statin use and other factors associated with mortality after major lower extremity amputation. J Vasc Surg 2017;66:216-225.

25. Kulik A, Shrank WH, Levin R, et al. Adherence to statin therapy in elderly patients after hospitalization for coronary revascularization. Am J Cardiol 2011;107:1409-1414.

26. O'Donnell TFX, Deery SE, Darling JD, et al. Adherence to lipid management guidelines is associated with lower mortality and major adverse limb events in patients undergoing revascularization for chronic limb-threatening ischemia. J Vasc Surg 2017;66:572-578. 
27. Stavroulakis K, Borowski M, Torsello G, et al. Association between statin therapy and amputation-free survival in patients with critical limb ischemia in the CRITISCH registry. J Vasc Surg 2017;66:1534-1542.

28. McTaggart F, Jones P. Effects of statins on high-density lipoproteins: a potential contribution to cardiovascular benefit. Cardiovasc Drugs Ther 2008;22:321-338.

29. Ferrara A, Barrett-Connor E, Shan J. Total, LDL, and HDL cholesterol decrease with age in older men and women. The Rancho Bernardo Study 1984-1994. Circulation 1997;96:37-43. 30. Lu M, Lu Q, Zhang Y, Tian G. ApoB/apoA1 is an effective predictor of coronary heart disease risk in overweight and obesity. J Biomed Res 2011;25:266-273.

31. Oksala N, Seppälä I, Hernesniemi J, et al. Complementary prediction of cardiovascular events by estimated apo- and lipoprotein concentrations in the working age population. The Health 2000 Study. Ann Med 2013;45:141-148.

32. van Capelleveen JC, Bochem AE, Boekholdt SM, et al. Association of high-density lipoprotein-cholesterol versus apolipoprotein A-I with risk of coronary heart disease: The European Prospective Investigation Into Cancer-Norfolk Prospective Population Study, the Atherosclerosis Risk in Communities Study, and the Women's Health Study. J Am Heart Assoc 2017;6:e006636.

33. Boekholdt SM, Arsenault BJ, Hovingh GK, et al. Levels and changes of HDL cholesterol and apolipoprotein A-I in relation to risk of cardiovascular events among statin-treated patients: a meta-analysis. Circulation 2013;128:1504-1512.

34. Pol T, Held C, Westerbergh J, et al. Dyslipidemia and risk of cardiovascular events in patients with atrial fibrillation treated with oral anticoagulation therapy: Insights from the ARISTOTLE (Apizaban for Reduction in Stroke and Other Thromboembolic Events in Atrial Fibrillation) Trial. J Am Heart Assoc 2018;7:e007444.

35. Ahnström J, Gottsäter A, Lindblad B, et al. Plasma concentrations of apolipoproteins A-I, B, and $M$ in patients with critical limb ischemia. Clin Biochem 2010;43:599-603.

36. Skinner JS, Farrer M, Albers CJ, et al. High apolipoprotein AI concentrations are associated with lower mortality and myocardial infarction five years after coronary artery bypass graft surgery. Heart 1999;81:488-494. 